\title{
Antiproton Cooling in the Fermilab Recycler Ring ${ }^{1}$
}

\author{
S. Nagaitsev ${ }^{\mathrm{a} 2}$, A. Bolshakov ${ }^{\mathrm{b}}$, D. Broemmelsiek ${ }^{\mathrm{a}}$, A. Burov ${ }^{\mathrm{a}}$, \\ K. Carlson ${ }^{\mathrm{a}}$, C. Gattuso ${ }^{\mathrm{a}}$, M. Hu ${ }^{\mathrm{a}}$, G. Kazakevich ${ }^{\mathrm{c}}$, B. Kramper ${ }^{\mathrm{a}}$, \\ T. Kroc ${ }^{\mathrm{a}}$, J. Leibfritz ${ }^{\mathrm{a}}$, L. Prost ${ }^{\mathrm{a}}, \mathrm{S}$. Pruss ${ }^{\mathrm{a}}$, G. Saewert ${ }^{\mathrm{a}}$, \\ C.W. Schmidt ${ }^{\mathrm{a}}$, S. Seletskiy ${ }^{\mathrm{d}}$, A. Shemyakin ${ }^{\mathrm{a}}$, M. Sutherland ${ }^{\mathrm{a}}$, \\ V. Tupikov ${ }^{\mathrm{a}}$, A. Warner ${ }^{\mathrm{a}}$, and P. Zenkevich ${ }^{\mathrm{b}}$ \\ ${ }^{a}$ FNAL, Batavia, IL 60510, U.S.A.; \\ ${ }^{b}$ ITEP, Moscow, 117259, Russia; \\ ${ }^{c}$ Budker INP, Novosibirsk, 630090, Russia; \\ ${ }^{d}$ University of Rochester, Rochester, NY 14627, U.S.A.
}

\begin{abstract}
The 8.9-GeV/c Recycler antiproton storage ring is equipped with both stochastic and electron cooling systems. These cooling systems are designed to assist accumulation of antiprotons for the Tevatron collider operations. In this paper we report on an experimental demonstration of electron cooling of high-energy antiprotons. At the time of writing this report, the Recycler electron cooling system is routinely used in collider operations. It has helped to set recent peak luminosity records.
\end{abstract}

Keywords: beam cooling, antiprotons, storage ring

PACS: 29.27.Eg, 29.27.Fh, 29.20.Dh, 41.75.-i, 41.85.Ew

\section{INTRODUCTION}

The Run II Luminosity Upgrade Plan at Fermilab requires the Recycler [1] to play a key role as the repository of large stacks of antiprotons $\left(6 \times 10^{12}\right)$ with the appropriate phase space characteristics to be used in the Tevatron collider stores. In order to maximize the stacking efficiency of the Fermilab antiproton Accumulator, small stacks of antiprotons will be frequently (every $0.5-1$ hour) transferred to the Recycler. In the Recycler, the stacks are initially cooled by stochastic cooling [2] and then stored and cooled by electron cooling until the antiprotons are ready to be used in the Tevatron.

The Run II Luminosity Upgrade Plan foresees the Recycler fully integrated into collider operations in two major steps. First, the Recycler is commissioned to bring its performance to the level that it is ready to begin the implementation of electron cooling. This milestone was achieved on June 1, 2004. In the second phase, the installation of electron cooling and its commissioning takes place. Electron cooling was demonstrated on July 15, 2005. At the time of writing this report the electron

1 Work supported by the URA, Inc., under contract DE-AC02-76CH03000 with the U.S. Dept. of Energy.

${ }^{2}$ E-mail: nsergei@fnal.gov 
cooling system is used for each Tevatron store. However, frequent antiproton transfers have not been implemented yet.

This paper outlines the design parameters of the Recycler Electron Cooling System and then describes the steps that led to the demonstration of electron cooling.

\section{COOLING SYSTEM PARAMETERS}

\section{System Layout}

Electron cooling of $8.9-\mathrm{GeV} / \mathrm{c}$ antiprotons in the Recycler requires an electron beam with kinetic energy of $4.3 \mathrm{MeV}$ [3]. Figure 1 shows the schematic layout of the Recycler electron cooling system.

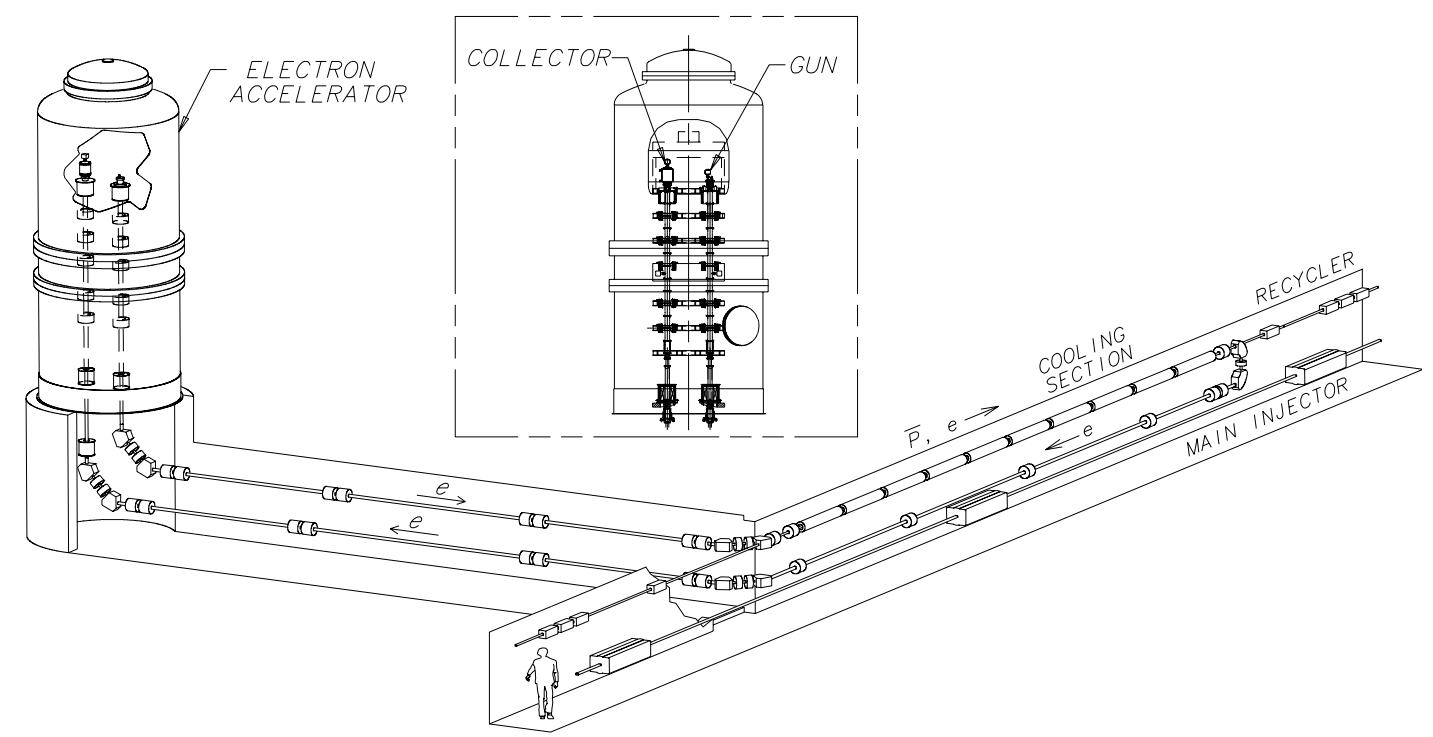

FIGURE 1. Schematic layout of the Recycler electron cooling system and accelerator cross-section (inset).

The dc electron beam is generated by a thermionic-cathode gun, located in the highvoltage (HV) terminal of the electrostatic (Van-de-Graaff type) accelerator. This accelerator is incapable of sustaining dc beam currents to ground in excess of about $100 \mu \mathrm{A}$. To attain the electron dc current of $500 \mathrm{~mA}$, a recirculation scheme is employed. The electron beam is first delivered to the cooling section and then returned back to the HV terminal for charge recovery. A typical inefficiency of such a process is $20 \mathrm{ppm}$ for beam currents of up to $500 \mathrm{~mA}$.

The electron cooling system at Fermilab employs a unique beam transport scheme [4]. The electron gun is immersed in a solenoidal magnetic field, which creates a beam with large angular momentum. After the beam is extracted from the magnetic field and accelerated to $4.3 \mathrm{MeV}$, it is transported to the $20-\mathrm{m}$ long cooling section solenoid using conventional focusing elements. At the entrance to the cooling section solenoid the beam is made round and parallel such that the beam radius, $r_{b}$, produces the same magnetic flux, $\mathrm{Br}_{r}{ }^{2}$, as at the cathode. 
Table 1 presents basic parameters of the Recycler ring and its electron cooling system.

TABLE 1. Electron cooling system and Recycler ring design parameters.

\begin{tabular}{lccc}
\hline Parameter & Symbol & Value & Units \\
\hline & Electron Accelerator & & \\
\hline Terminal Voltage & $U_{0}$ & 4.34 & $\mathrm{MV}$ \\
Beam Current & $I_{b}$ & 0.5 & $\mathrm{~A}$ \\
Terminal Voltage Ripple, rms & $\delta U$ & 200 & $\mathrm{~V}$ \\
\hline \multicolumn{4}{c}{ Cooling Section } \\
\hline Length & $\mathrm{L}$ & 20 & $\mathrm{~m}$ \\
Solenoid Field & $B$ & 100 & $\mathrm{G}$ \\
Beam Radius & $r_{b}$ & 3.5 & $\mathrm{~mm}$ \\
Electron Angular Spread, rms & $\theta_{e}$ & $\leq 0.2$ & $\mathrm{mrad}$ \\
\hline \multicolumn{4}{c}{ Recycler design parameters } \\
\hline Circumference & $C$ & 3.3 & $\mathrm{~km}$ \\
Momentum & $\beta \gamma M c$ & 8.9 & $\mathrm{GeV} / \mathrm{c}$ \\
Transition $\gamma$ & $\gamma_{t}$ & 20.7 & \\
Ave. beta functions & $\beta_{a v e}$ & 30 & $\mathrm{~m}$ \\
Typical emittance (n, 95\%) & $\varepsilon$ & $5-7$ & $\mu \mathrm{m}-\mathrm{rad}$ \\
Number of antiprotons & $N_{a}$ & $\leq 600$ & $10^{10}$ \\
Average pressure & $P_{a v}$ & 0.5 & $\mathrm{nTorr}$ \\
\hline
\end{tabular}

\section{Cooling Scenario}

The electron cooling scenario has been reviewed in Ref. [5]. Under the current scenario the electron cooling system is required to decrease the longitudinal $95 \%$ emittance of a stored antiproton beam from $100 \mathrm{eV}$-s to $50 \mathrm{eV}$-s in 30 minutes for stacks of up to $6 \times 10^{12}$ particles. This would correspond to providing 36 equally populated bunches with a $1.5-\mathrm{eV}-\mathrm{s}$ longitudinal emittance per bunch to the Tevatron collider.

Figure 2 presents a MOCAC code [6] simulation of the electron cooling process in the Recycler with $6 \times 10^{12}$ antiprotons in a barrier rf bucket. The process of cooling is optimized by keeping the rms antiproton momentum spread constant at $3.5 \mathrm{MeV} / \mathrm{c}$, while continuously reducing the bunch length by moving the rf barriers. The initial transverse emittance in this simulation was $5 \mu \mathrm{m}$-rad. The three curves correspond to different values of the electron beam rms angular spread in the cooling section. The design value of $0.2 \mathrm{mrad}$ for the rms angular spread is presented by the bottom curve. Other electron beam parameters are presented in Table 1.

The choice of the rms momentum spread being $3.5 \mathrm{MeV} / \mathrm{c}$ is determined by a compromise between the IBS-related longitudinal diffusion and the beam lifetime due to the dynamic momentum aperture of the Recycler ring. Figure 3 shows the calculated longitudinal and transverse IBS growth rates as a function of the rms momentum spread. One can see that for the momentum spread of $3.5 \mathrm{MeV} / \mathrm{c}$ the longitudinal diffusion is practically independent of the transverse emittance. 


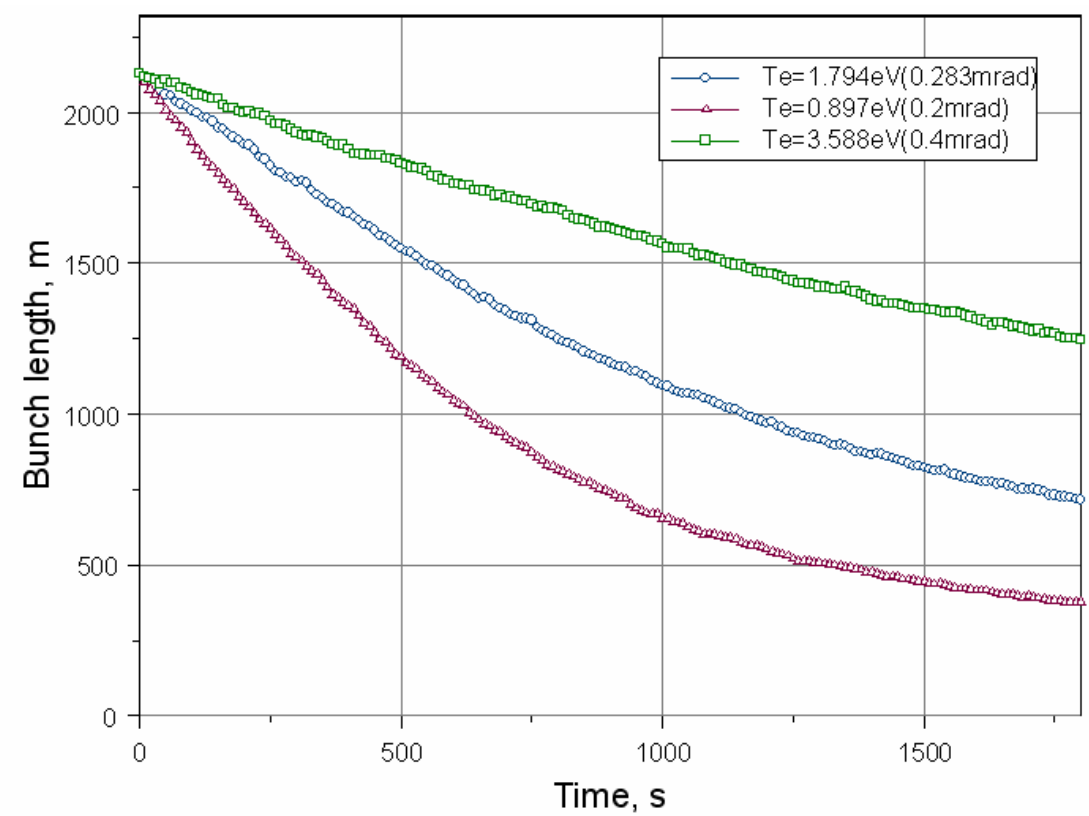

FIGURE 2. The evolution (simulation) of the antiproton bunch length as a function of time (30 minutes full scale) for various electron beam angular spreads. The initial bunch length corresponds to a $100 \mathrm{eV}$-s longitudinal emittance. The design curve (bottom) indicates that there is a factor of two safety margin in cooling rates.
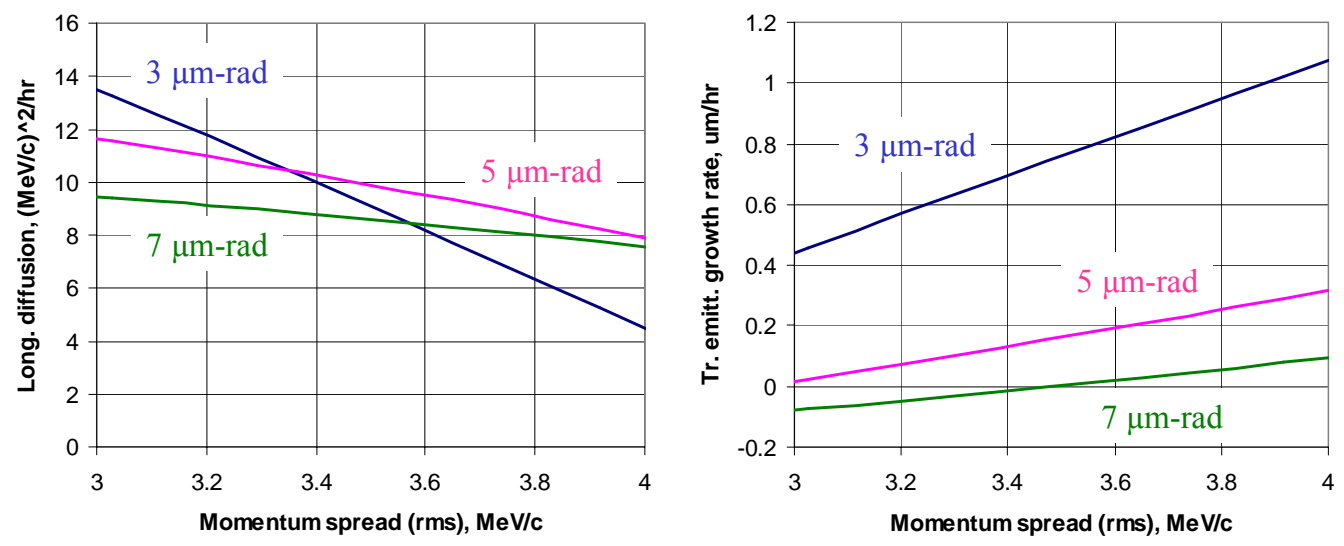

FIGURE 3. The simulated IBS heating rates (Bjorken-Mtingwa model) for $250 \times 10^{10}$ antiprotons with a constant $54 \mathrm{eV}$-s longitudinal emittance as a function of the rms momentum spread for various transverse emittances.

\section{COOLING DEMONSTRATION}

\section{Energy Alignment}

It has been anticipated that in the early stages of commissioning, the electron cooling system might not be able to meet three major design parameters concurrently: (1) the beam current of $0.5 \mathrm{~A}$, (2) the rms angular spread of $0.2 \mathrm{mrad}$ and (3) the 
perfect energy matching between electron and antiproton beams. We have also anticipated that for the electron beam current and angular spread optimization it would be highly desirable to see the "effect" of cooling first. Since the cooling process is quite slow and the electron energy was not known to the required accuracy, we estimated that the energy matching could be challenging. Our plan for energy matching was based on two assumptions: (1) the Recycler absolute energy is known to $0.1 \%$ and (2) the Recycler momentum acceptance is greater than $0.3 \%$. Measuring and adjusting the electron absolute energy to $0.3 \%$ [7] would allow us to "land" the electron beam energy somewhere within the Recycler momentum acceptance and to observe the cooling effect even for lower beam currents and higher angular spreads. To observe the cooling process we implemented the following procedure. A small $\left(<10 \times 10^{10}\right)$ antiproton beam current was debunched and cooled transversely (by the stochastic cooling system) to a small transverse beam emittance. Using an rf noise source the momentum spread of this beam was increased to create a uniform momentum distribution $0.3-0.4 \%$ wide. The antiproton-electron beam interaction was observed with the help of a longitudinal beam Schottky-noise monitor, which measures the momentum distribution function. Figure 4 presents a simulation of this process.

The simulation in Fig. 4 was performed with a coasting antiproton beam perfectly matched in energy with the electron beam of $0.1 \mathrm{~A}$ and with $0.5 \mathrm{mrad}$ of rms angular spread. The spike in the distribution, formed by the electron cooling process, increases the distribution function by a factor of 2 - a value easily detectable by a Schottky-noise spectrum analyzer.

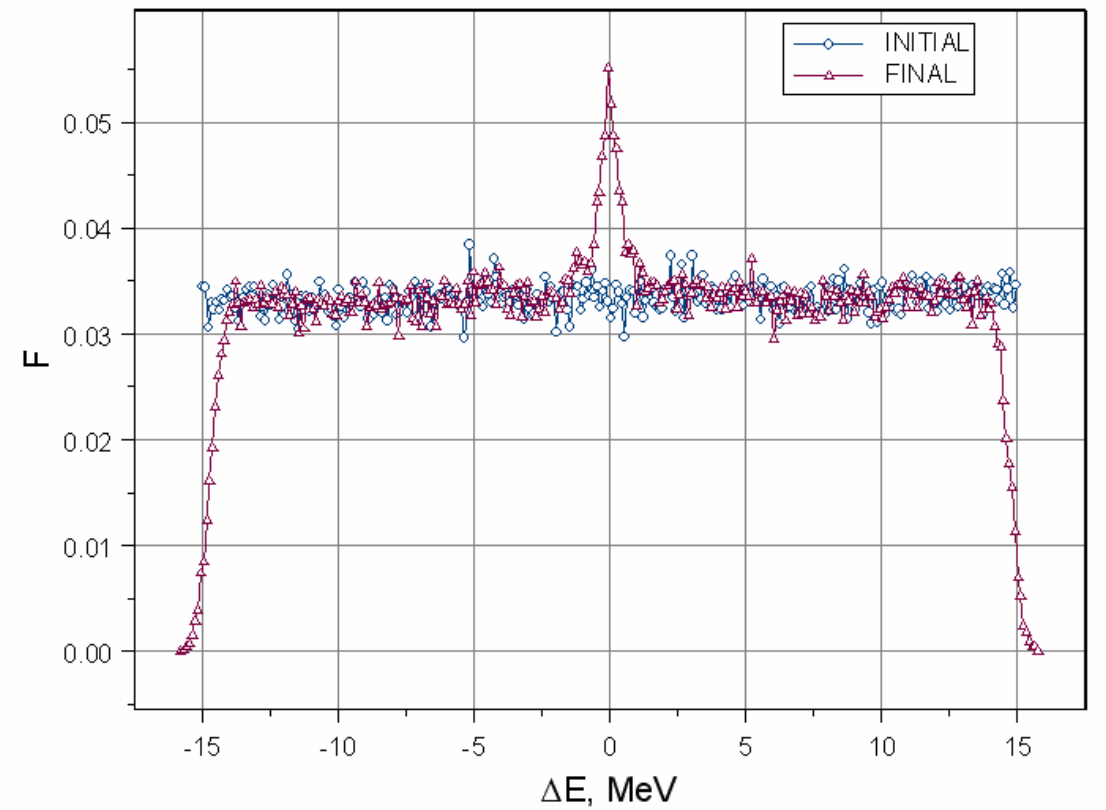

FIGURE 4. The momentum distribution (arb. units) as a function of antiproton energy deviation (simulation by MOCAC code [5]). The initial distribution is uniform in energy. The final distribution is plotted after 30 minutes.

Using this method, the initial observations of cooling in the Recycler demonstrated that the electron energy was within $3 \mathrm{keV}$ of its optimal value. Figure 5 shows the 
experimental implementation of this procedure in the Recycler after the electron energy adjustment to its optimal value.

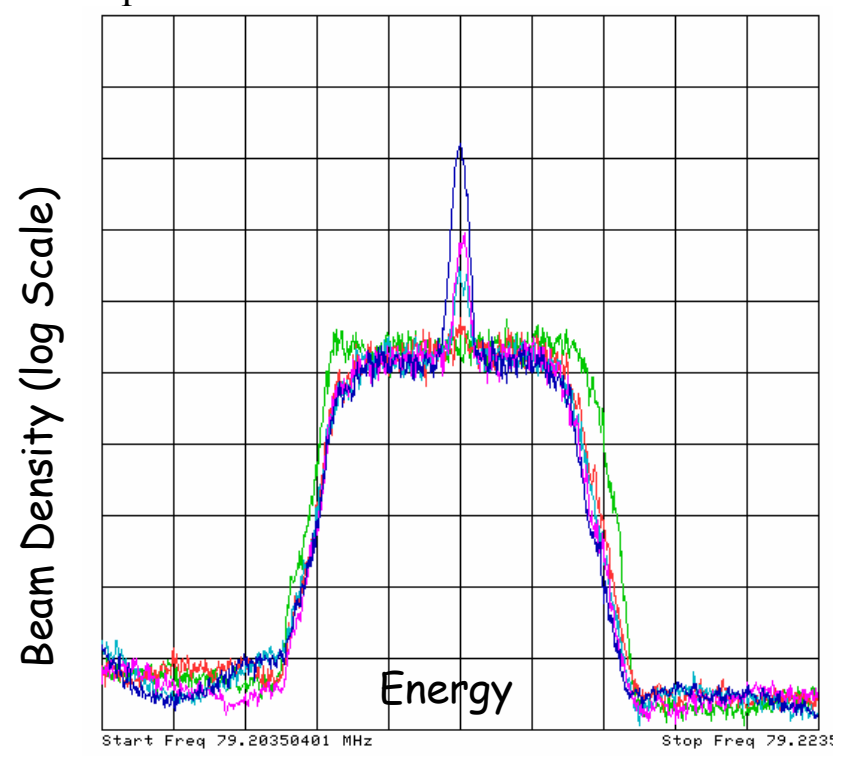

FIGURE 5. The evolution of the antiproton distribution function with electron cooling. The horizontal scale is $25 \mathrm{MeV} / \mathrm{div}$. The antiproton beam intensity was $5 \times 10^{10}$ and its emittance was $2 \mu \mathrm{m}$-rad. Traces were taken 15 minutes apart.

\section{Cooling Observation}

The first electron cooling demonstration was performed on July 15, 2005 with a bunched beam of $63 \times 10^{10}$ antiprotons. Figure 6 shows two antiproton momentum distribution functions, taken 15 minutes apart, while the beam was electron cooled.

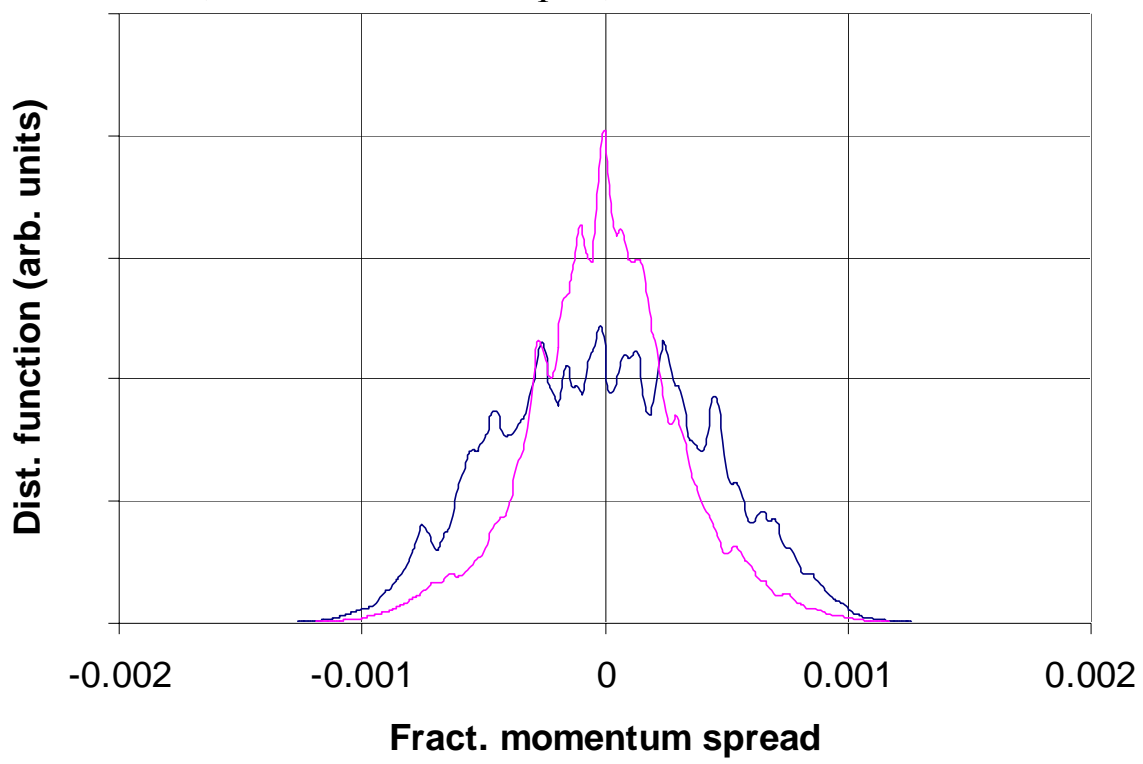

FIGURE 6. The first cooling demonstration of relativistic antiprotons in the Recycler. The antiproton current was $63 \times 10^{10}$, the emittance was $4 \mu \mathrm{m}$-rad, and the bunch length was $1.7 \mu \mathrm{s}$. The electron beam current was $200 \mathrm{~mA}$. Traces were taken 15 minutes apart. 
To measure the cooling force we employed the so-called voltage jump method [8]. In this method, the coasting antiproton beam is initially cooled down to a small equilibrium momentum spread. The electron beam energy is then changed instantaneously by several $\mathrm{keV}$. The electron cooling force then drags the antiproton distribution to a new equilibrium momentum, which is $M / m$ times the voltage jump away from the initial equilibrium. Figure 7 presents the evolution of the antiproton momentum distribution function as the antiprotons are being dragged by the electron beam after its energy was jumped by $2 \mathrm{keV}$. The initial average cooling force, determined from this plot, is about $20 \mathrm{MeV} / \mathrm{c}$ per hour - this agrees well with our theoretical predictions.

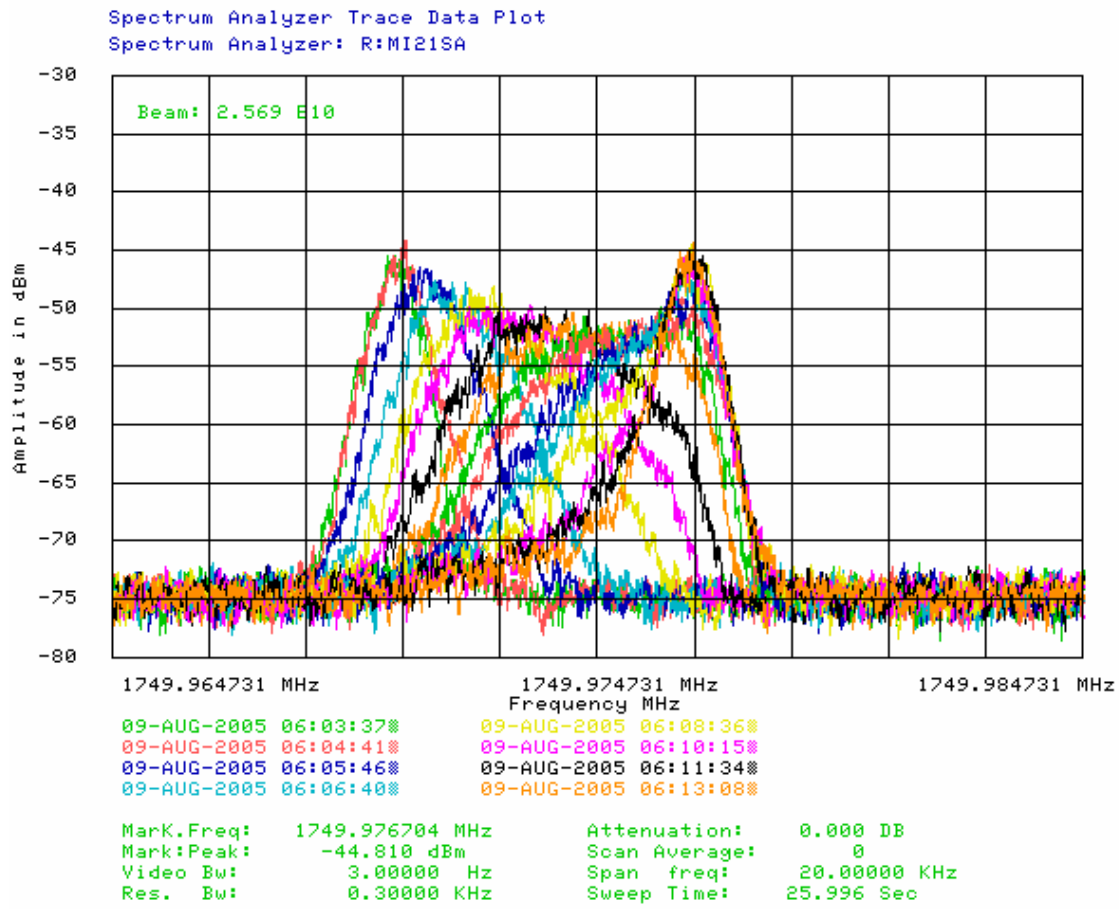

FIGURE 7. The evolution of the antiproton beam momentum distribution function as the antiprotons are being dragged by a 200-mA electron beam to a new equilibrium after the energy jump. Left curve the initial distribution and right - new equilibrium after the energy jump. Traces are taken approximately 1 minute apart. The number of antiprotons was $4 \times 10^{10}$, the transverse emittance was 1.5 $\mu \mathrm{m}-\mathrm{rad}(\mathrm{n}, 95 \%)$. The horizontal scale is $1.2 \mathrm{MeV} / \mathrm{c}$ per division.

Finally, Figure 8 presents the evolution of both the transverse and the longitudinal emittances of the antiproton beam, being cooled by the electron cooling system. Our initial estimates show that the electron cooling rates are high enough to meet the final requirements of the Run II luminosity upgrades as outlined above. 


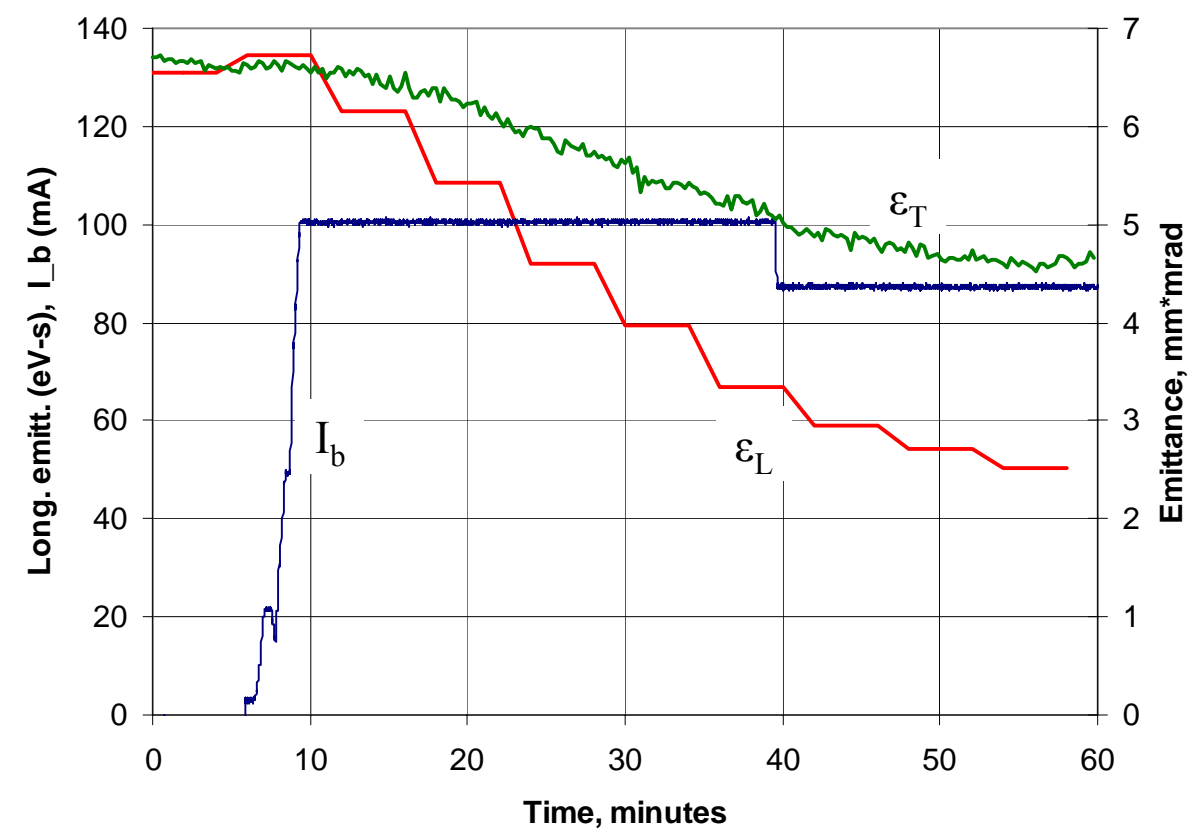

FIGURE 8. Electron cooling of a bunched antiproton beam $\left(78 \times 10^{10}\right)$. The electron beam was turned on to $100 \mathrm{~mA}$ at 10 minutes. The step-like variation of the longitudinal emittance is due to the extended interval between consecutive data points.

\section{SUMMARY}

Electron cooling of $8-\mathrm{GeV}$ antiprotons has been demonstrated and is presently in routine operations. The measured cooling force is in agreement with theoretical predictions. The electron cooling system has been used in Tevatron collider operations since August, 2005. Since then, it has been primarily responsible for the recent advances in the Tevatron peak luminosity.

\section{ACKNOWLEDGMENTS}

The authors are grateful to Fermilab's technical staff for installing and maintaining the cooling system and to A.C. Crawford, D. Prasuhn, V. Lebedev, I.N. Meshkov, V.V. Parkhomchuk, and V. Reva for their help and for many useful discussions. We acknowledge contributions made by R. Goodwin, J. Crisp, and L. Carmichael to the protection system; by L. Nobrega and S. Wesseln to the vacuum system design; and by B. Chase and P. Joireman to the design of the BPM system and to beam oscillation measurements.

\section{REFERENCES}

1. G. Jackson, FERMILAB-TM-1991 (1996).

2. D. Broemmelsiek, M. Hu and S. Nagaitsev, "Stochastic cooling in barrier buckets at the Fermilab Recycler", EPAC'04, Lucerne, July 2004. 
3. "Prospectus for an electron cooling system for the Recycler," edited by J.A. MacLachlan, FERMILAB-TM2061, Oct 1998.

4. A. Burov, et al., Phys. Rev. Sp. Top. - AB 3, 094002 (2000).

5. A. Burov, "Electron-cooling scenarios at Fermilab", NIM A 532 (2004) 291-297.

6. P. R. Zenkevich et al., "Modeling of Electron Cooling by Monte Carlo Method", Report at the International Workshop on Cooling and Related Topics, Bad Honnef (Germany), 2001.

7. S. M. Seletskiy and A. Shemyakin, Beam-based calibration of the electron energy in the Fermilab electron cooler, to be published in Proc. of PAC'05, Knoxville, USA, May 16-20, 2005

8. H. Danared, et al., Phys. Rev. Lett. 72, 3775 (1994). 